\title{
$m^{\text {th }}$-ORDER FISHER-KPP EQUATION WITH FREE BOUNDARIES AND A GENERAL ADVECTION
}

\author{
CHANGQING JI, DANDAN ZHU, AND JINGLI REN
}

\begin{abstract}
In this paper, we investigate a $m$ th-order Fisher-KPP equation with free boundaries and a general advection term. Considering the influence of advection term and initial conditions on the long time behaviour of solutions, we obtain spreading-vanishing dichotomy, spreading-transition-vanishing trichotomy, and vanishing happens with the coefficient of advection term in small amplitude, medium sized amplitude and large amplitude, respectively. Then, the appropriate parameters are selected in the simulation to intuitively show the corresponding theoretical results. Moreover, the wave-spreading and wave-vanishing are observed in our study.
\end{abstract}

\section{INTRODUCTION}

Reaction-diffusion equation is widely used to investigate various phenomena in physics, chemistry and biology. The equation with the form of $u_{t}=u_{x x}+f(t, x, u)$ can be used to describe the spread of biological population or chemical substances etc $[1,4,9,10,12,19]$. Li et al. [11] explored species spread in the context of climate change by considering this model with $f(t, x, u)=u r(x-c t)-u^{2}$. Hu and Zou [8] considered the traveling wave solutions and predicted the speed and manner of a biological species will die out when $f(u)$ is a generalized Fisher-KPP type of nonlinearity with a shifting habitat.

In reality, the expansion of biological populations are also often affected by advection; in the mean time, a biological species typically lives in a bounded domain with the boundary moving/expanding according some rules. These suggest incorporation of advection into a reaction-diffusion equation on a bounded domain with moving boundary. It is with these considerations, that the following free boundary equation has been proposed and studied for the 1-dimension space:

$$
u_{t}=u_{x x}-\beta(t) u_{x}+f(t, x, u), t>0, g(t)<x<h(t) .
$$

When $\beta(t)$ is a constant, equation (1.1) can be used to describe the dynamic behaviour of biological population in the case of constant coefficient advection. Gu et al. $[5,6]$ proved the spreading-vanishing dichotomy by considering this equation with small advection environment and Fisher-KPP type of nonlinearity. When $\beta(t)=\beta$ is constant and $f(t, x, u)=f(u)$ is homogeneous in time and space and $f(u)$ is nonlinearity of Fisher type, Gu et al. [7] studied this equation with free boundaries and obtained the long time behaviour of the solutions. Ren and Zhu [16] investigated this equation when $\beta(t)=\beta$ is constant and $f(t, x, u)=f(u)$ is a $m^{t h}$-order Fisher-KPP type and obtained the following results: (i) for small advection (small $\beta$ ), there is the spreading-transition-vanishing trichotomy; (ii) for median-sized advection, there is the virtual spreading-transition-vanishing trichotomy; (iii) for large advection, there is the vanishing dynamics.

Received by the editors 20 May 2021; accepted 23 July 2021; published online 31 July 2021.

2010 Mathematics Subject Classification. 35C07, 35R35, 35K57.

Key words and phrases. $m^{\text {th }}$-order Fisher-KPP equation, general advection term, long time behaviour. 
When $f(t, x, u)=f(t, u)$ and both $\beta(t)$ and $f(t, u)$ are both T-periodic in time variable $t$, equation (1.1) can be used to describe the biological population dynamics in time-periodic environment. The equation with a periodic Fisher-KPP type of nonlinearity has been studied widely by many scholars $[2,13,14,17]$. Sun et al. [18] proposed a T-periodic advection-reaction-diffusion problem with free boundaries. They obtained the following results: (i) there is a spreading-vanishing dichotomy in the case of $\bar{\beta} \in[0, \bar{c})$; (ii) there is a spreading-transition-vanishing trichotomy in the case of $\bar{\beta} \in[\bar{c}, \mathcal{B}(\tilde{\beta})$ ); (iii) vanishing happens in the case of $\bar{\beta} \geq \mathcal{B}(\tilde{\beta})$. Here

$$
\bar{c}=2 \sqrt{\frac{1}{T} \int_{0}^{T} f_{u}(t, 0) d t}, \quad \bar{\beta}=\frac{1}{T} \int_{0}^{T} \beta(t) d t,
$$

and $\tilde{\beta}(t)=\beta(t)-\bar{\beta}, \mathcal{B}(\tilde{\beta})$ is a function depending on $\tilde{\beta}(t)$.

Motivated by the above works, it is worth to study the equation where the advection term is timedependent but not necessarily periodic, and the reaction term is a general $m^{\text {th }}$-order Fisher-KPP type of nonlinearity. For convenience, we consider a special time-dependent advection coefficient, that is, an oscillatory coefficient with time varying amplitude: $\beta(t)=\lambda(t) \sin (t)$, which will not be periodic if $\lambda(t)$ is not a periodic function, or if it is a periodic but the period is different from $2 \pi$. The specific free boundary system as follows:

$$
\left\{\begin{array}{l}
u_{t}=u_{x x}-\lambda(t) \sin (t) u_{x}+\alpha u^{m}(1-u), t>0, g(t)<x<h(t), \\
g^{\prime}(t)=-\mu u_{x}(t, g(t)), u(t, g(t))=0, t>0, \\
h^{\prime}(t)=-\mu u_{x}(t, h(t)), u(t, h(t))=0, t>0, \\
-g(0)=h(0)=h_{0}, u(0, x)=u_{0}(x), \quad-h_{0} \leq x \leq h_{0},
\end{array}\right.
$$

where $\mu$ and $\alpha$ are positive constants, $m \in \mathbb{N}^{*}$. The initial function $u_{0}(x) \in \Phi\left(h_{0}\right)$ for $h_{0}>0$, where

$\Phi\left(h_{0}\right)=\left\{\phi \in C^{2}\left(\left[-h_{0}, h_{0}\right]\right): \phi\left(-h_{0}\right)=\phi\left(h_{0}\right)=0, \phi^{\prime}\left(-h_{0}\right)>0, \phi^{\prime}\left(h_{0}\right)<0, \phi(x)>0\right.$ for $\left.x \in\left(-h_{0}, h_{0}\right)\right\}$.

System (1.2) contains a number of advection-reaction-diffusion equations. For example, it is the FisherKpp equation with time-periodic advection when $\lambda(t)$ is a constant, $m=1[17,18]$.

Suppose that

$$
\lambda(t) \in C^{\gamma / 2}(\mathbb{R})(\gamma \in(0,1)) \text {, and } \lambda(t) \sin (t) \text { is an integrable function. }
$$

In this paper, we first give the global existence and uniqueness of the solution. Then, by considering the influence of advection coefficient and initial value conditions on the solutions, we obtain a spreadingvanishing dichotomy with small amplitude of $\beta(t)$; a spreading-transition-vanishing trichotomy with medium-sized amplitude of $\beta(t)$; vanishing happens with large amplitude of $\beta(t)$. The partition of $\beta(t)$ relies on the mean value

$$
\bar{\beta}:=\frac{1}{2 \pi} \int_{k \pi}^{k \pi+2 \pi} \beta(t) d t
$$

and the shape $\tilde{\beta}(t):=\beta(t)-\bar{\beta}$. In addition, we find some new phenomena in numerical simulations, i.e. wave-spreading and wave-vanishing cases. Now, we give the following concepts:

(i) Wave-Spreading: $I_{\infty}=\mathbb{R}, \lim _{t \rightarrow \infty} u(t, \cdot)=1$ locally uniformly in $\mathbb{R}$ and the solution $u$ oscillates left and right in the spreading domain;

(ii) Wave-Vanishing: $I_{\infty}$ is a finite length interval, $\lim _{t \rightarrow \infty} \max _{g(t) \leq x \leq h(t)} u(t, x)=0$ and the solution $u$ oscillates left and right in the vanishing domain,

where

$$
I(t):=[g(t), h(t)], g_{\infty}:=\lim _{t \rightarrow \infty} g(t), h_{\infty}:=\lim _{t \rightarrow \infty} h(t) \text { and } I_{\infty}:=\left(g_{\infty}, h_{\infty}\right) .
$$


This paper is structured as follows. In Section 2, we present the global existence and uniqueness of the solution. Long time behaviour of the solutions are obtained with different 'sizes' of a general function $\lambda(t)$ in Section 3. To show the theoretical results more intuitively, we give some numerical simulations in Section 4.

\section{Global existence and uniqueness}

In this section, we first establish the local existence and uniqueness of the solution, and then show that the solution exists globally and is unique. To this end, we need to recall the following conditions on a general nonlinearity function $f(t, x, u)$ required in [20]:

$\left(H_{1}\right) f$ is a Lipschitz continuous function respect to $x$ : For any given $\tau, l, k>0$, there exists a constant $M_{1}(\tau, l, k)$ such that

$$
\left|f\left(t, x_{1}, u\right)-f\left(t, x_{2}, u\right)\right| \leq M_{1}(\tau, l, k)\left|x_{1}-x_{2}\right| \text { for }\left(t, x_{i}, u\right) \in(0, \tau) \times(0, l) \times(0, k), i=1,2 .
$$

$\left(H_{2}\right) f$ is a Lipschitz continuous function respect to $u$ : For any given $\tau, l, k>0, f \in L^{\infty}((0, \tau) \times$ $(0, l) \times(0, k))$ and there exists a constant $M_{2}(\tau, l, k)$ such that

$$
\left|f\left(t, x, u_{1}\right)-f\left(t, x, u_{2}\right)\right| \leq M_{2}(\tau, l, k)\left|u_{1}-u_{2}\right| \text { for }\left(t, x, u_{i}\right) \in(0, \tau) \times(0, l) \times(0, k), i=1,2 .
$$

In system (1.2), since reaction term $f(u):=\alpha u^{m}(1-u x . S i)\left(m \in \mathbb{N}^{*}\right)$ independent of $t$ and $x$, condition $\left(H_{1}\right)$ obviously holds. $\left(H_{2}\right)$ can also be easily verified for this nonlinear function $f(u)$. Thus, we can use the results in [20] to establish the following lemma.

Lemma 2.1. There exists a $T>0$ such that problem (1.2) has a unique solution $(u, g, h)$ in $[0, T]$, which satisfies

(i) $g(t), h(t) \in C^{1+\gamma / 2}([0, T]), u \in W_{p}^{1,2}\left(G_{T}\right) \cap C^{(1+\gamma) / 2,1+\gamma}\left(\bar{G}_{T}\right)$;

(ii) $0<-g^{\prime}(t), h^{\prime}(t)<L_{1}, 0 \leq u(t, x) \leq L_{2},(t, x) \in G_{T}$,

where $G_{T}:=\{(t, x):(t, x) \in(0, T) \times(g(t), h(t))\}$, the constants $L_{1}, L_{2}$ and $T$ only depend on $h_{0}, \alpha$, and $\left\|u_{0}\right\|_{C^{2}\left(\left[-h_{0}, h_{0}\right]\right)}$.

Proof. Consider the following transformation

$$
(t, x) \rightarrow(t, y), \text { where } x=y+\zeta(-y)\left(g(t)+h_{0}\right)+\zeta(y)\left(h(t)-h_{0}\right), y \in \mathbb{R} .
$$

Let

$$
\zeta(y)=\frac{e^{-\left(y-h_{0}\right)^{2}}-e^{-4 h_{0}^{2}}}{1-e^{-4 h_{0}^{2}}}
$$

we have $\zeta(y) \in C^{\infty}(\mathbb{R}), \zeta\left(-h_{0}\right)=0, \zeta\left(h_{0}\right)=1$ and

$$
\zeta^{\prime}(y)=\frac{-2\left(y-h_{0}\right) e^{-\left(y-h_{0}\right)^{2}}}{1-e^{-4 h_{0}^{2}}} .
$$

Since $\zeta^{\prime}(y) \in C(\mathbb{R})$ and $\lim _{y \rightarrow \pm \infty} \zeta^{\prime}(y)=0$, there exists $L>0$, such that

$$
\zeta^{\prime}(y)<\frac{L}{h_{0}} .
$$

The transformation is a differential homeomorphism as long as

$$
\max \left\{\left|h(t)-h_{0}\right|,\left|g(t)+h_{0}\right|\right\} \leq \frac{h_{0}}{2 L}
$$


is satisfied. Then, the free boundaries $x=g(t)$ and $x=h(t)$ are transformed to $y=-h_{0}$ and $y=h_{0}$ respectively. Due to the properties of $\beta(t)$ and $f(u)$, using the similar arguments in the proof of Theorem 1.1 in [20], we obtain that there exists a $T>0$ such that (1.2) has a unique solution $(u, g, h)$ and satisfies

$$
g(t), h(t) \in C^{1+\gamma / 2}([0, T]), u \in W_{p}^{1,2}\left(G_{T}\right) \cap C^{(1+\gamma) / 2,1+\gamma}\left(\bar{G}_{T}\right) .
$$

Based on the properties of $\beta(t), u_{0}(x)$ and $f(u)$, we may argue as the proof of Lemma 2.2 in [3] to complete the estimates of $(u, g, h)$.

Lemma 2.2. Assume the conditions in Lemma 2.1 hold, then problem (1.2) has a unique global solution.

Proof. Denote

$$
T_{0}=\sup \left\{T>0:(u, g, h) \in C\left(\bar{G}_{T}\right) \times C^{1}([0, T]) \times C^{1}([0, T]) \text { is the solution of }(1.2)\right\},
$$

where $T_{0}$ is called the maximal existence time of the solution of problem (1.2). Combining with the idea of Theorem 1.2 in [20], we have $T_{0}=\infty$. This means that the global existence and uniqueness of the solution are obtained.

\section{LONG TIME BEHAVIOR OF THE SOLUTIONS}

Combining with the conditions in (1.3) and the conclusions in $[7,18]$, the following system

$$
\begin{cases}u_{t}=u_{x x}-\lambda(t) \sin (t) u_{x}+\alpha u^{m}(1-u), & t, x \in \mathbb{R}, \\ u(t,-\infty)=0, u(t, \infty)=1, u(0,0)=\frac{1}{2}, & t \in \mathbb{R}, \\ u(t+2 k \pi, x)=u(t, x+X)=1, & t, x \in \mathbb{R}, X:=(\bar{c}-\bar{\beta}),\end{cases}
$$

has a traveling wave solution $u:=Q\left(t, x+\bar{c} t-\int_{0}^{t} \lambda(s) \sin (s) d s\right)$, which can be further expressed as $Q(t, x+(\bar{c}-\bar{\beta}) t)$, where

$$
\bar{c}=2 \sqrt{\frac{1}{2 \pi} \int_{0}^{2 \pi} f_{u}(t, 0) d t}, \bar{\beta}=\frac{1}{2 \pi} \int_{k \pi}^{k \pi+2 \pi} \lambda(t) \sin (t) d t \quad(k \in \mathbb{N})
$$

are critical values to partition the small and medium-sized advection. Denote $\tilde{\beta}(t):=\lambda(t) \sin (t)-\bar{\beta}$, from the research of subsection 3.3 and 3.4 in [18], we obtain that there exists a function $B(\tilde{\beta})$, which is depends on $\tilde{\beta}(t)$, such that $B(\tilde{\beta})$ is a critical value function to partition the medium-sized and the large advection.

\subsection{Vanishing case.}

Assume $|\bar{\beta}|<\bar{c}$ and (1.3) are established. According to Lemma 3.1 in [17], the following vanishing conditions are equivalent:

(i) $I_{\infty}$ is a finite interval;

(ii) $h_{\infty} \leq h^{*}(-\beta(t), a(t))$, where $h^{*}(-\beta(t), a(t))$ is defined as Lemma 2.1 in [17];

(iii) $\lim _{t \rightarrow \infty}\|u(t, \cdot)\|_{L^{\infty}\left(\left[-h_{0}, h_{0}\right]\right)}=0$.

This means that $h_{\infty}<\infty$ is a necessary and sufficient condition for vanishing. Therefore, we have the following lemma.

Lemma 3.1. Assume $|\bar{\beta}|<\bar{c}$ and (1.3) are established. Then vanishing happens if the following initial conditions hold

(i) $h_{0}<h^{*}(-\beta(t), a(t))$;

(ii) $\left\|u_{0}\right\|_{L^{\infty}\left(\left[-h_{0}, h_{0}\right]\right)}$ is sufficiently small. 
Proof. Since $h_{0}<h^{*}(-\beta(t), a(t))$, choose $h_{1} \in\left(h_{0}, h^{*}(-\beta(t), a(t))\right)$ and consider the problem

$$
\begin{cases}\mathcal{L} \omega:=\omega_{t}-\omega_{z z}+\beta(t) \omega_{z}-a(t) \omega=\kappa \omega, & t \geq 0,0<z<h_{1}, \\ \omega(t, 0)=0, \omega\left(t, h_{1}\right)=0, & t \geq 0 \\ \omega(0, z)=\omega(k \pi+2 \pi, z), & 0 \leq z \leq h_{1}\end{cases}
$$

Denote $\kappa_{1}$ is the principal eigenvalue of (3.2), $\omega_{1}>0$ is the corresponding eigenfunction and $\left\|\omega_{1}\right\|_{L^{\infty}}=1$. We have $\kappa_{1}>0$ by using Lemma 2.1 in [17]. We use $\xi_{1}(t)$ and $\xi_{2}(t)$ to denote the left most and right most local maximum point of $w_{1}(t, \cdot)$, respectively. Set

$$
\begin{gathered}
\varepsilon_{1}:=\min _{t \geq 0} \xi_{1}(t), \varepsilon_{2}:=\max _{t \geq 0} \xi_{2}(t), \\
\eta_{0}:=\min \left\{\min _{t \geq 0} \omega_{1}\left(t, \varepsilon_{1}\right), \min _{t \geq 0} \omega_{1}\left(t, \varepsilon_{2}\right)\right\}, \delta:=\min \left\{\kappa_{1}, \frac{h_{1}}{h_{0}}-1\right\},
\end{gathered}
$$

then $\eta_{0} \leq 1$, and there exists small $\eta_{1}=\eta(\delta)$ such that

$$
\eta_{1} \cdot \max \left\{\max _{t \geq 0}\left|\mu \omega_{1 x}\left(t, h_{1}\right)\right|, \max _{t \geq 0}\left|\mu \omega_{1 x}(t, 0)\right|\right\}<\frac{\delta^{2}}{2} h_{0} .
$$

Denote

$$
w(t, x):=\eta_{0} \eta_{1} e^{-\delta t} \omega_{1}\left(t, x+h_{1}\right),(t, x) \in[0, \infty) \times\left[-h_{1}, h_{1}\right],
$$

then for the properties of $f(t, \omega)$,

$$
\begin{aligned}
& w_{t}-w_{x x}+\beta(t) w_{x}-f(t, w) \\
= & -\delta \eta_{0} \eta_{1} e^{-\delta t} \omega_{1}\left(t, x+h_{1}\right)+\omega_{t}-\omega_{z z}+\beta(t) \omega_{z}-a(t) \omega \\
\geq & \eta_{0} \eta_{1} e^{-\delta t}\left(-\delta+\kappa_{1}\right) \omega_{1}\left(t, x+h_{1}\right) \\
\geq & 0 .
\end{aligned}
$$

Since $w(0, x)=\eta_{0} \eta_{1} \omega_{1}\left(0, x+h_{1}\right)$, we choose the initial function $u_{0}(x)$ satisfies

$$
u_{0}(x) \leq \eta_{0} \eta_{1} \omega_{1}\left(0, x+h_{1}\right), x \in\left[-h_{0}, h_{0}\right],
$$

by the Lemma 3.7 in [2], we obtain

$$
u(t, x) \leq \omega(t, x),(t, x) \in[0, \tau) \times\left[-h_{0}, h_{0}\right],
$$

where $\tau:=\sup \left\{t>0: g(t)>-h_{1}, h(t)<h_{1}\right\}$. According to the proof of Lemma 3.3 in [17], we have $\tau=\infty$. This implies

$$
[g(t), h(t)] \subset\left[-h_{1}, h_{1}\right], t \geq 0,
$$

i.e. $I_{\infty}$ is a finite interval. Therefore, vanishing happens.

Similarly, choose $h_{2}>h_{0}$, and denote $\kappa_{2}$ is the principal eigenvalue of (3.2), $\omega_{2}>0$ with $\left\|\omega_{2}\right\|_{L^{\infty}}=1$ is the corresponding eigenfunction. Since $\bar{\beta} \geq \bar{c}$, then $\kappa_{2}>0$. We have the following lemma.

Lemma 3.2. Assume $\bar{c} \leq \bar{\beta}<B(\tilde{\beta})$ and (1.3) are established. Let $(u, g, h)$ be the solution of (1.3). Then vanishing happens if $\left\|u_{0}\right\|_{L^{\infty}\left(\left[-h_{0}, h_{0}\right]\right)}$ is sufficiently small. 


\subsection{Spreading case.}

Lemma 3.3. Assume $\bar{c} \leq \bar{\beta}<B(\tilde{\beta})$ and (1.3) are established. Let $(u, g, h)$ be the solution of (1.3). Then spreading happens if there exist $x_{1}, L_{c}$ such that

$$
u(t, x) \geq W_{L_{c}}\left(0, x-x_{1}\right) \text { for } x \in\left[x_{1}-L_{c}, x_{1}\right],
$$

where $0<c \ll 1, W_{L_{c}}$ is the compactly supported traveling wave defined in [18].

Proof. Since

$$
\beta(t) \in C^{\gamma / 2}([0, \infty)) \text { for } \gamma \in(0,1), \beta(k \pi)=\beta(k \pi+2 \pi)=0, k \in \mathbb{N}^{+} .
$$

For any small $L_{c}>0$, when $x_{1}$ is large enough, using proposition 3.16 in [18], system (1.2) has a low solution

$$
u=W_{L_{c}}:=U_{0}\left(t, R_{L_{c}}-x, \beta-R_{L_{c}}, x_{1}\right),
$$

where $R_{L_{c}}=\int_{0}^{t} r_{L_{c}}(s) d s, r(t, \beta)=-\mu u_{x}(t, R(t, \beta)), r_{L_{c}}=r(t, \beta)-L_{c}, 0<\bar{r}<\bar{\beta}+\bar{c}$. Thus, we can prove this lemma by similar arguments in [18].

\subsection{Sharp threshold.}

For any given initial value $h_{0}>0$ and $\phi \in \Phi\left(h_{0}\right)$, denote $(u(t, x ; \sigma \phi), g(t ; \sigma \phi), h(t ; \sigma \phi)):=(u, g, h)$ be the solution of (1.2) with $u_{0}=\sigma \phi$. For convenience, set

$$
\begin{aligned}
& \Omega_{1}:=\{\sigma \geq 0 \mid \text { vanishing happens for } u(\cdot, \cdot ; \sigma \phi)\}, \sigma_{*}:=\sup \Omega_{1}, \\
& \Omega_{2}:=\{\sigma \geq 0 \mid \text { spreading happens for } u(\cdot, \cdot ; \sigma \phi)\}, \sigma^{*}:=\inf \Omega_{2} .
\end{aligned}
$$

According to Lemma 3.1 and Lemma 3.2, we obtain $\sigma \in \Omega_{1}$ for all small $\sigma>0$, namely $\Omega_{1} \neq \emptyset$.

Theorem 3.4. Assume $0 \leq \bar{\beta}<\bar{c}$ and (1.3) are established, $(u, g, h)$ is a solution of (1.2) with $u_{0}=\sigma \phi$ for $\phi \in \Phi\left(h_{0}\right)$. Then, there exist $\sigma_{*}=\sigma_{*}\left(h_{0}, \phi, \beta\right), \sigma^{*}=\sigma^{*}\left(h_{0}, \phi, \beta\right)$ such that $\sigma_{*}=\sigma^{*}$ and

(i) if $\sigma \in\left[0, \sigma_{*}\right]$, vanishing happens;

(ii) if $\sigma \in\left(\sigma^{*}, \infty\right)$, spreading happens.

Proof. Obviously, if $\sigma_{*}=\infty$, only vanishing happens. Thus, in the following proof, we assume that $\sigma_{*} \in(0, \infty)$. Now, we prove $\sigma_{*}=\sigma^{*}$. Otherwise, if $\sigma_{*}<\sigma^{*}$, there exist $\sigma_{1}, \sigma_{2} \in\left(\sigma_{*}, \sigma^{*}\right)$ with $\sigma_{1}<\sigma_{2}$. Using the comparison principle, we have

$$
\begin{gathered}
g\left(t ; \sigma_{2} \phi\right)<g\left(t ; \sigma_{1} \phi\right), h\left(t ; \sigma_{1} \phi\right)<h\left(t ; \sigma_{2} \phi\right), \\
u\left(t, x ; \sigma_{2} \phi\right)<u\left(t, x ; \sigma_{1} \phi\right) \text { for }(t, x) \in(0, \infty) \times\left(g\left(t ; \sigma_{1} \phi\right), h\left(t ; \sigma_{1} \phi\right)\right) .
\end{gathered}
$$

Based on the continuous dependence of the solution $u(t, x ; \sigma \phi)$ with respect to $x$, there exists $\delta_{1}>0$ such that

$$
u\left(1, x ; \sigma_{1} \phi\right)<u\left(1, x-\delta_{1} ; \sigma_{2} \phi\right) \text { for } x \in\left[g\left(t ; \sigma_{1} \phi\right), h\left(t ; \sigma_{1} \phi\right)\right] .
$$

Using the comparison principle again, we obtain

$$
u\left(t, x ; \sigma_{1} \phi\right)<u\left(t, x-\delta_{1} ; \sigma_{2} \phi\right) \text { for }(t, x) \in(1, \infty) \times\left(g\left(t ; \sigma_{1} \phi\right), h\left(t ; \sigma_{1} \phi\right)\right) .
$$

Thus, when $(t, x) \in(1, \infty) \times\left[g\left(t ; \sigma_{1} \phi\right)-h\left(t ; \sigma_{2} \phi\right), 0\right]$, we have

$$
u\left(t, x+h\left(t ; \sigma_{1} \phi\right) ; \sigma_{1} \phi\right)<u\left(t, x+h\left(t ; \sigma_{2} \phi\right)+\lambda(t)-\delta_{1} ; \sigma_{2} \phi\right),
$$

where $\lambda(t)=h\left(t ; \sigma_{1} \phi\right)-h\left(t ; \sigma_{2} \phi\right)$. By using Lemma 4.1 in [18], we have $u\left(t, x+h\left(t ; \sigma_{1} \phi\right) ; \sigma_{1} \phi\right)$ and $u\left(t, x+h\left(t ; \sigma_{2} \phi\right) ; \sigma_{2} \phi\right)$ converges to 0 uniformly, that is, $\lim _{t \rightarrow \infty} \lambda(t)-\delta_{1}=0$. This is a contradiction. Thus, $\sigma_{*}=\sigma^{*}$.

Next, we show that vanishing happens when $\sigma=\sigma_{*}$. If not, spreading happens when $\sigma=\sigma_{*}$, and there exists $t_{0}>0$ such that $h\left(t_{0}\right)-g\left(t_{0}\right)>h^{*}(-\beta(t), a(t))+1$. By the continuous dependence of 
$(u(t, x ; \sigma \phi), g(t ; \sigma \phi), h(t ; \sigma \phi))$, taking $\epsilon>0$ is sufficiently small, denote $\left(u_{\epsilon}(t, x ; \sigma \phi), g_{\epsilon}(t ; \sigma \phi), h_{\epsilon}(t ; \sigma \phi)\right)$ is a solution of (1.2) with $u_{0}=\left(\sigma_{*}-\epsilon\right) \phi$, and satisfies

$$
h_{\epsilon}\left(t_{0}\right)-g_{\epsilon}\left(t_{0}\right)>h^{*}(-\beta(t), a(t)) .
$$

According to Lemma 3.4 in [17], spreading happens for the solution $\left(u_{\epsilon}(t, x ; \sigma \phi), g_{\epsilon}(t ; \sigma \phi), h_{\epsilon}(t ; \sigma \phi)\right)$ with $u_{0}=\left(\sigma_{*}-\epsilon\right) \phi$. It is a contradiction with the definition of $\sigma_{*}$. Thus, vanishing happens when $\sigma=\sigma_{*}$.

Theorem 3.5. Assume $\bar{c} \leq \bar{\beta}<B(\tilde{\beta})$ and (1.3) are established, $(u, g, h)$ is a solution of (1.2) with $u_{0}=\sigma \phi$ for $\phi \in \Phi\left(h_{0}\right)$. Then there exist $\sigma_{*}=\sigma_{*}\left(h_{0}, \phi, \beta\right)$ and $\sigma^{*}=\sigma^{*}\left(h_{0}, \phi, \beta\right)$ such that

(i) if $\sigma \in\left[0, \sigma_{*}\right)$, vanishing happens;

(ii) if $\sigma \in\left[\sigma_{*}, \sigma^{*}\right]$, transition happens: $g_{\infty}>-\infty, h_{\infty}=+\infty, \lim _{t \rightarrow \infty} u(t, \cdot)=0$ locally uniformly in $I_{\infty}$ and spreading does not happen;

(iii) if $\sigma \in\left(\sigma^{*}, \infty\right)$, spreading happens.

Proof. From previous illustration, $\Omega_{1} \neq \emptyset$ and $\left[0, \sigma_{*}\right) \in \Omega_{1}$. In the following, we assume $\sigma_{*} \in(0, \infty)$ (if $\sigma_{*}=\infty$, only vanishing happens). According to Theorem 4.9 in [7], $\Omega_{1}=\left[0, \sigma_{*}\right)$. Moreover, when $\sigma \geq \sigma_{*}, g_{\infty}>-\infty, h_{\infty}=+\infty, \lim _{t \rightarrow \infty} u(t, \cdot)=0$ locally uniformly in $\left(g_{\infty},+\infty\right)$.

On the other hand, if $\Omega_{2}=\emptyset$, spreading does not happen for all $\sigma \geq 0$. Thus transition happens when $\sigma \geq \sigma_{*}$. If $\Omega_{2}=\emptyset$, that is $\sigma^{*}<\infty$. From Lemma 3.3 and the continuous dependence of $(u(t, x ; \sigma \phi), g(t ; \sigma \phi), h(t ; \sigma \phi)), \Omega_{2}$ is an open set. Thus $\Omega_{2}=\left[\sigma^{*}, \infty\right)$.

According to the above analysis, neither spreading nor vanishing happens for $u(t, x ; \sigma \phi)$ with $\sigma \in$ $\left[\sigma_{*}, \sigma^{*}\right]$. Therefore, if $\sigma \in\left[\sigma_{*}, \sigma^{*}\right], u\left(t, x ; \sigma_{*} \phi\right)$ is a transition solution and

$$
g_{\infty}>-\infty, h_{\infty}=+\infty, \lim _{t \rightarrow \infty} u(t, \cdot)=0 \text { locally uniformly in }\left(g_{\infty},+\infty\right) .
$$

Theorem 3.6. Assume $\bar{\beta} \leq-\bar{c}$ or $\bar{\beta} \geq B(\tilde{\beta})$ and (1.3) are established. $(u, g, h)$ is a time-global solution of (1.2) with $u_{0}=\sigma \phi$ for $\phi \in \Phi\left(h_{0}\right)$. Then vanishing happens.

Proof. According to Lemma 3.7 and Lemma 3.8 in [17], we obtain

$$
h_{\infty}<\infty, \lim _{t \rightarrow \infty}\|u(t, \cdot)\|_{L^{\infty}([g(t), h(t)])}=0 .
$$

This means vanishing happens.

\section{Numerical Simulation AND ANALYsis}

In order to show the theoretical results more intuitively, we use the similarly scheme in [15] to give the numerical analysis of system (1.2) in this section. Assume

$$
\alpha=3, \mu=1, h_{0}=5, \phi=\cos \left(\frac{\pi}{10} x\right) .
$$

According to the properties of $\beta(t)$, we choose $\beta(t)=\rho t \sin t$, then

$$
\begin{aligned}
& \bar{\beta}=\frac{1}{2 \pi} \int_{k \pi}^{k \pi+2 \pi} \rho t \sin t d t=-\rho \cos k \pi, \\
& \tilde{\beta}=\rho t \sin t+\rho \cos k \pi, k=1,2,3, \cdots
\end{aligned}
$$

Thus, the asymptotic behaviour of the solutions to system (1.2) will be determined by the amplitude $\rho$ and initial value $\sigma$. 
Example 4.1. Fig.1(a) is the numerical result with $m=3, \rho=0.01, \sigma=0.1$. The free boundaries $x=g(t)$ and $x=h(t)$ increase slowly, and the solution decays to zero with the increase of time $t$. In this case, vanishing happens, which corresponding to $\beta(t)$ is a small function case in Theorem 3.4(i). Fig.2(a) shows the curve of $u(t, \cdot)$ at $t=5,15,30$. Obviously, with the increase of time $t$, this curve tends to zero. Fig.1(b) is the numerical result with $m=3, \rho=0.01, \sigma=1$. The two free boundaries increase faster than in Fig.1(a) and the solution stabilizes to 1 with the increase of time $t$. This means spreading happens, which corresponding to $\beta(t)$ is a small function case in Theorem 3.4(ii). When spreading occurs, Fig.2(b) shows the curve of $u(t, \cdot)$ at $t=10,20,30$, we can see that $u(t, \cdot)$ has a maximum value of 1 near $x=0$. Besides, with the increase of time $t$, the spread domain of $u(t, \cdot)$ with respect to $x$ also expands.

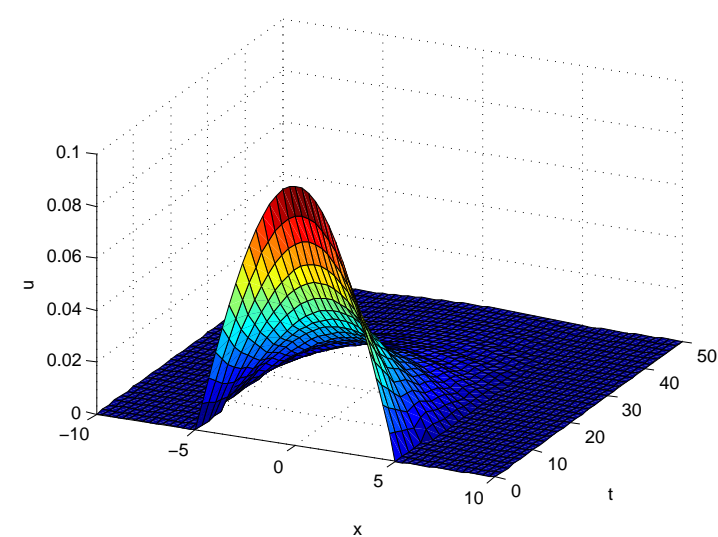

(a)

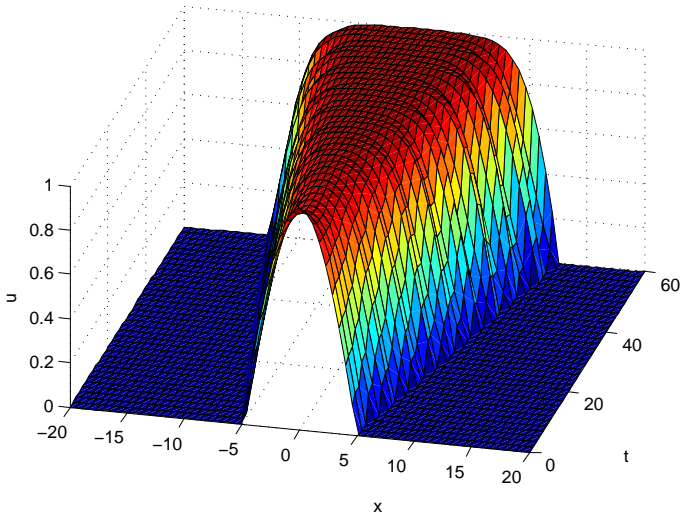

(b)

Figure 1. Numerical simulation results of system (1.2) when $m=3, \rho=0.01$. (a): $\sigma=0.1 ;(\mathrm{b}): \sigma=1$.

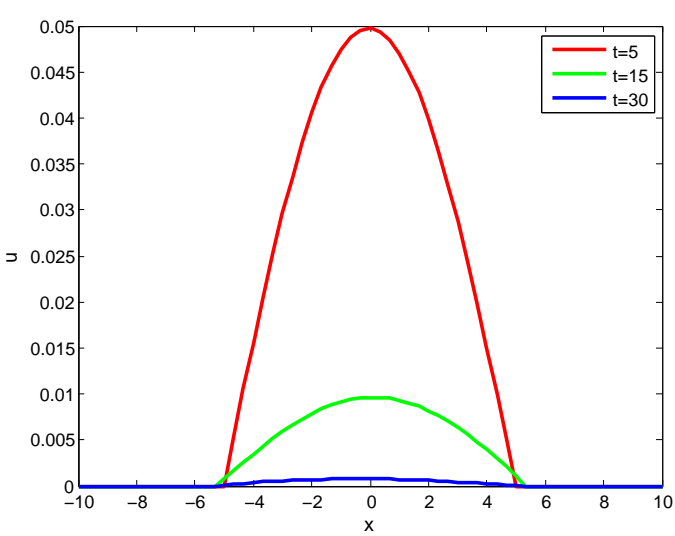

(a)

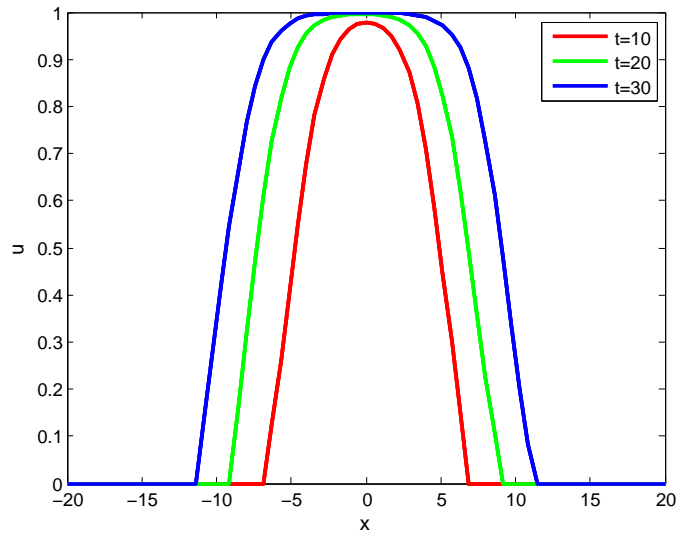

(b)

Figure 2. The curve of $u(t, \cdot)$ at different $t$. (a): vanishing happens; (b): spreading happens. 
Example 4.2. Fig.3(a) is the numerical result for $m=3, \rho=0.1, \sigma=0.1$. The free boundaries rises in waves slowly, and the solution decays to zero with the increase of time $t$. In this case, vanishing happens (we can also call it wave-vanishing, since the free boundaries rises in waves), which corresponding to $\beta(t)$ is a medium-sized function case in Theorem 3.5(i). Fig.4(a) shows the curve of $u(t, \cdot)$ at $t=5,15,30$. Similarly with Fig.2(a), the curve $u(t, \cdot)$ tends to zero. Fig.3(b) is the numerical result for $m=3$, $\rho=0.1, \sigma=1$. It can be seen from Fig.3(b) that free boundaries $x=g(t)$ and $x=h(t)$ rises in waves faster than in Fig.3(a). Besides, the solution converges locally to 1 with the increase of time $t$. This implies spreading happens (we can also call it wave-spreading since the free boundaries rises in waves), which corresponding to $\beta(t)$ is a medium-sized function case in Theorem 3.5(ii). Fig.4(b) shows the curve of $u(t, \cdot)$ at $t=13,20,28$. Comparing the curves at different times, we can also find that the solution spreading in waves.

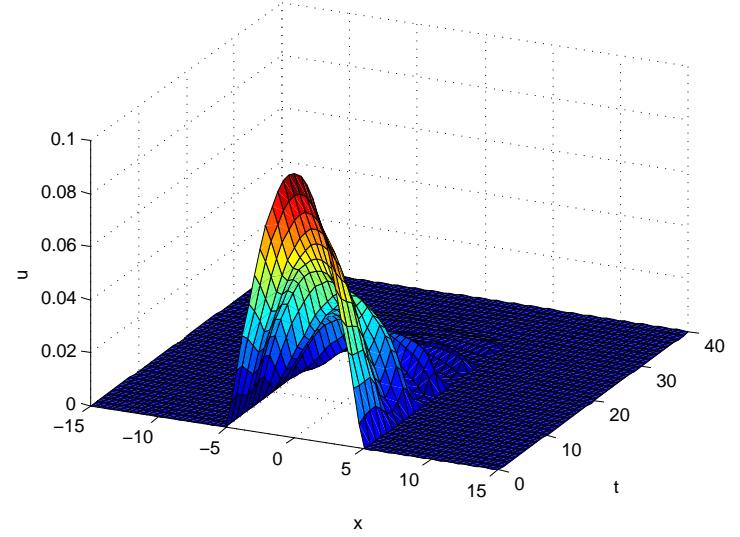

(a)

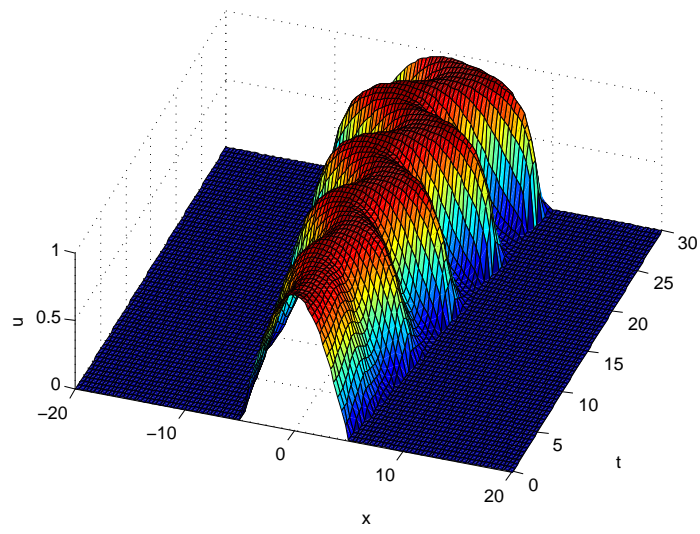

(b)

Figure 3. Numerical simulation results of system (1.2) when $m=3, \rho=0.1$. (a): $\sigma=0.1 ;(\mathrm{b}): \sigma=1$.

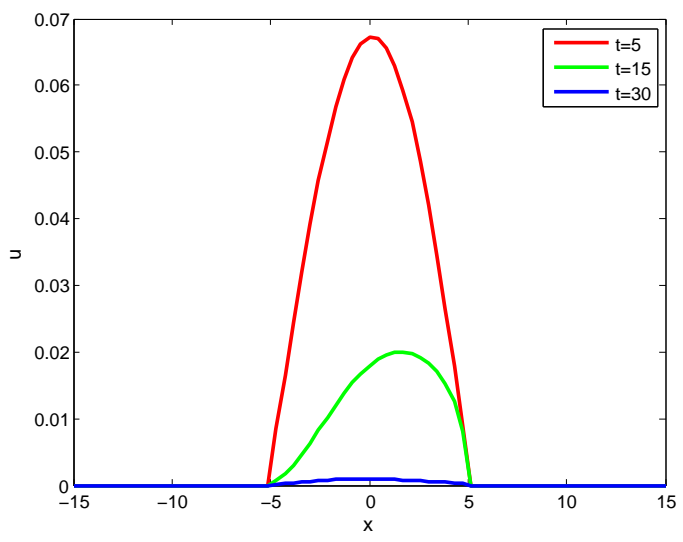

(a)

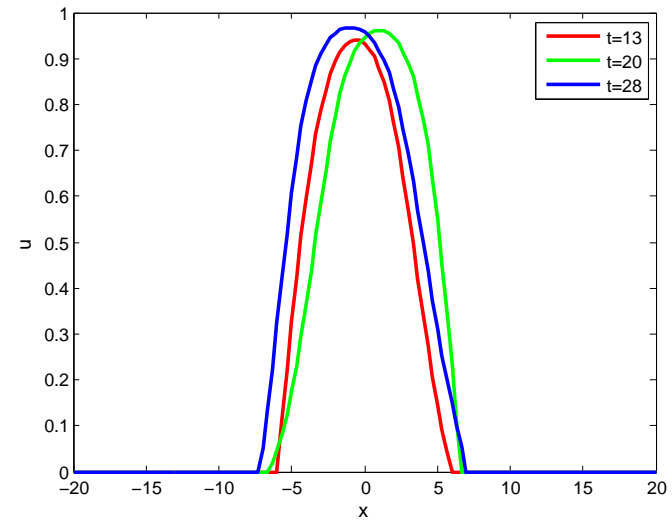

(b)

Figure 4. The curve of $u(t, \cdot)$ at different $t$. (a): vanishing happens; (b): spreading happens. 
Example 4.3. Let $m=3, \rho=1$, The numerical results with $\sigma=0.1$ and $\sigma=1$ are shown in Fig.5(a) and Fig.5(b) respectively. From these two pictures, we find that the free boundaries rises in waves slowly, and the solution decays to zero quickly. Same as the previous analysis, we can also call this as wave vanishing case. Similarly, we obtain that wave vanishing happens in Fig.6(a) and Fig.6(b). These two cases corresponding to Theorem 3.1.

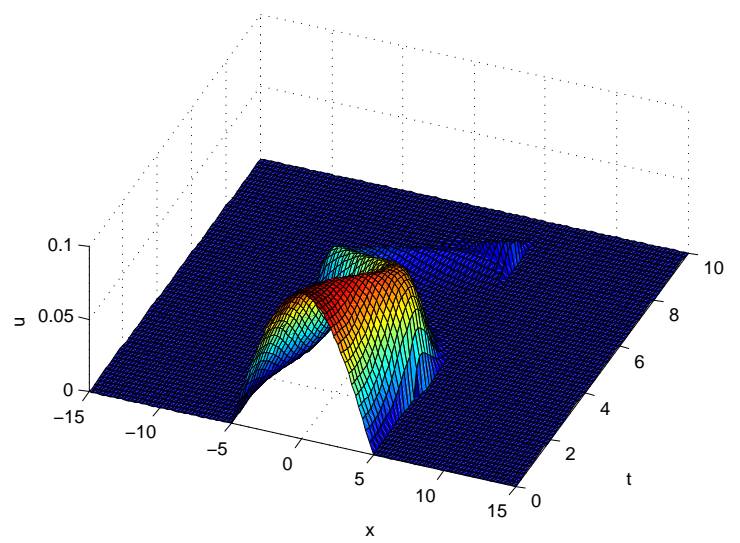

(a)

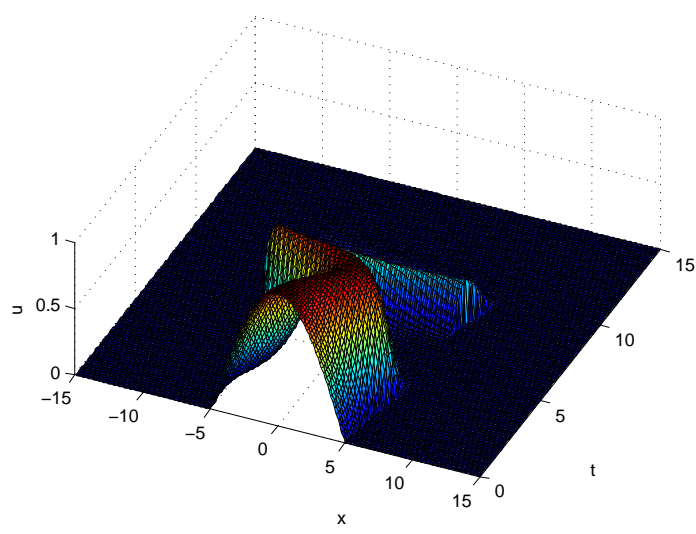

(b)

Figure 5. Numerical simulation results of system (1.2) when $m=3, \rho=1$. (a): $\sigma=$ $0.1 ;(\mathrm{b}): \sigma=1$.

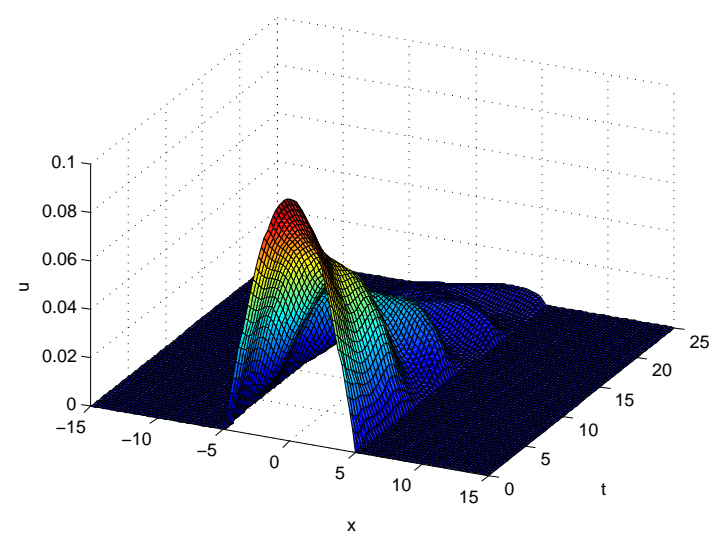

(a)

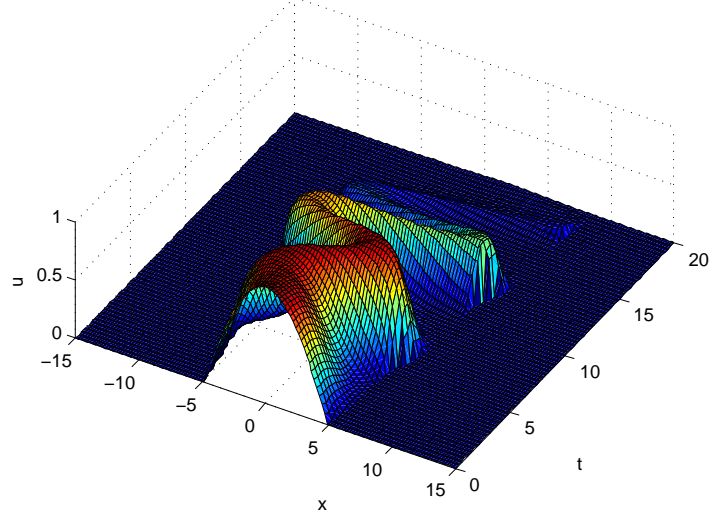

(b)

Figure 6. Numerical simulation results of system (1.2) when $m=3, \rho=-0.5$. (a): $\sigma=0.1 ;(\mathrm{b}): \sigma=1$.

\section{REFERENCES}

[1] X. F. Chen, B. D. Lou, M. L. Zhou and T. Giletti, Long time behaviour of solutions of a reaction diffusion equation on unbounded intervals with Robin boundary conditions, Ann. Inst. H. Poincar Anal. Non Linaire 33 (2016), 67-92. 
[2] Y. H. Du, Z. M. Guo and R. Peng, A diffusive logistic model with a free boundary in time-periodic environment, Journal of Functional Analysis 265 (2013), 2089-2142.

[3] Y. H. Du and Z. G. Lin, Spreading-vanishing dichotomy in the diffusive logistic model with a free boundary, SIAM Journal on Mathematical Analysis 42 (2010), 377-405.

[4] Y. H. Du and B. D. Lou, Spreading and vanishing in nonlinear diffusion problems with free boundaries, Journal of the European Mathematical Society 17 (2015), 2673-2724.

[5] H. Gu, Z. G. Lin and B. D. Lou, Different asymptotic spreading speeds induced by advection in a diffusion problem with free boundaries, Proceedings of the American Mathematical Society 143 (2015), 1109-1117.

[6] H. Gu, Z. G. Lin and B. D. Lou, Long time behaviour of solutions of a diffusion-advection logistic model with free boundaries, Applied Mathematics Letters 37 (2014), 49-53.

[7] H. Gu, B. D. Lou and M. L. Zhou, Long time behaviour of solutions of Fisher-KPP equation with advection and free boundaries, Journal of Functional Analysis 269 (2015), 1714-1768.

[8] H. J. Hu and X. F. Zou, Existence of an extinction wave in the Fisher equation with a shifting habitat, Proceedings of the American Mathematical Society 145 (2017), 4763-4711.

[9] H. J. Hu, T. S. Yi and X. F. Zou, On spatial-temporal dynamics of a Fisher-KPP equation with a shifting environment, Proceedings of the American Mathematical Society 148 (2020), 213-221.

[10] Y. Kaneko and Y. Yamada, A free boundary problem for a reaction-diffusion equation appearing in ecology, Advances in Mathematical Sciences and Applications 21 (2011), 467-492.

[11] B. T. Li, S. Bewick, J. Shang and W. F. Fagan, Erratum to: Persistence and Spread of A Species with A Shifting Habitat Edge. SIMA Journal on Applied Mathematics, 75 (2015), 2379-2380.

[12] X. D. Liu and B. D. Lou, Asymptotic behaviour of solutions to diffusion problems with Robin and free boundary conditions, Mathematical Modelling of Natural Phenomena 21 (2013), 18-32.

[13] Z. G. Lin, A free boundary problem for a predator-prey model, Nonlinearity 21 (2007), 1883-1892.

[14] G. Nadin, Existence and uniqueness of the solution of a space-time periodic reaction-diffusion equation, Journal of Differential Equations 249 (2010), 1288-1304.

[15] R. Stefănescu and G. Dimitriu, Numerical Approximation of a Free Boundary Problem for a Predator-Prey Model, Numerical Analysis \& Its Applications 5343 (2009), 548-555.

[16] J. L. Ren and D. D. Zhu, On a reactionadvectiondiffusion equation with double free boundaries and $m$ th-order Fisher non-linearity, IMA Journal of Applied Mathematics 84 (2019), 197-227.

[17] N. K. Sun, A time-periodic reaction-diffusion-advection equation with a free boundary and sign-changing coefficients, Nonlinear Analysis: Real World Applications 51 (2020), 102952.

[18] N. K. Sun, B. D. Lou and M. L. Zhou, Fisher-KPP equation with free boundaries and time-periodic advections, Calculus of Variations \& Partial Differential Equations 56 (2017), 61.

[19] H. F. Weinberger, Long-Time Behavior of a Class of Biological Models. SIAM Journal on Mathematical Analysis, 13 (1982), 353-396.

[20] M. X. Wang, Existence and uniqueness of solutions of free boundary problems in heterogeneous environments, Discrete \& Continuous Dynamical Systems 24 (2019), 415-421.

School of Mathematics and Statistics, Zhengzhou University, Zhengzhou 450001, China

E-mail address: jcq_115@163.com

School of Mathematics and Information Science, Zhengzhou University of Light Industry, Zhengzhou 450002, CHINA

E-mail address: zhudan@zzuli.edu.cn

Corresponding author, Henan Academy of Big Data, Zhengzhou University, Zhengzhou 450052, China

E-mail address: renj1@zzu.edu.cn 